\title{
Physical Informatics - Information Methods of Natural Systems Research
}

\author{
Igor Gurevich
}

iggurevich@gmail.com, Institute of Informatics Problems of the Russian Academy of Sciences, Hetnet Consulting Corp., Moscow, Russia

\begin{abstract}
The work confirms the priority of information laws, which are the basis of physical informatics: the information laws (informatics laws) are defined and restrict the physical laws; the informatics laws have a general, universal character, and operate in all possible universes with different physical laws. Physical Informatics is the science of modern Information in physical and chemical systems, including Quantum Informatics, and is the basis for Informatics of the Living Systems.
\end{abstract}

Keywords: Modern Informatics, Physical Informatics, Information (Informatics) Laws

Acknowledgement: The author thanks Academician K. Valiev, Academician N. Kardashev, Academician I. Sokolov, Professor S.Shorgin, Professor K. Colin, Dr. V. Sinitsyn, Dr. V. Chentsov, Professor V. Zhozhikashvili, Professor V. Lipunov, Dr. L. Gindilis, Dr. Abubukerov, Dr. D Panov, Dr. A. Leontovich, the company «GETNET Consulting» and its leader, M. Panin for interest in this area of work and assistance. I thank my wife, N. Solomentseva for support and assistance.

Along with matter and energy, the universe contains information. Information is an integral part of the universe. Each physical system has, along with physical characteristics, information characteristics. Information is inseparably linked with matter and energy. Scientists study physical characteristics and physical laws when they study information characteristics and information laws. This report provides an overview of the fundamental results obtained by the outstanding scientists Einstein (1935), Neumann (1932), Shannon (1948), Wheeler (1998), Penrose (1989), Brillouin (1960), Zeilinger (1999), Lloyd (2001), Lisi (2006), and Chernavsky (2002). The basic finding by the author is a formulation of nature's laws by means of more general than physical informatics laws (Gurevich, 1989-2010). The author conducted research of complex systems, including physical systems, in terms of informatics laws. The informatics laws define and limit physical phenomena and processes. The informatics laws precede the physical laws. The quantity of scientists using the information approach and information methods in physical research is quickly increasing.

\section{The List of the Main Results of the Author}

Information is physical heterogeneity. The information characteristics of heterogeneity are: Shannon's information entropy, information divergence, joint entropy, and communication information. The informatics laws of nature are: the law of simplicity of complex systems, the law of uncertainty (information) conservation, the law of finiteness of complex systems characteristics, the law of necessary variety by Ashby W. (1956), and the theorem of Gödel K. (1931). The main principle of quantum mechanics by Zeilinger (1999) is: elemental physical systems contain (carry) one bit of information. The law of finiteness of complex systems characteristics and the principle of necessary variety by Ashby impose restrictions on the topology and symmetry of the universe; time is onedimensional Euclidean space. Space is three-dimensional Euclidean space. Time is homogeneous. Space is homogeneous. Space is isotropic. Space is flat. The universe is four-dimensional pseudoEuclidean space. The law of simplicity of complex systems and the law of uncertainty (information) conservation impose restrictions on physical transformations of the space-time and transformation of internal symmetry: Jacobian transformations are equal to 1 . Transformations are linear. Equality to one of the determinants of linear transformation defines that, among the space-time transformations, only translations and owns rotations are physically possible. I Irreversibility of time, not the 
owns rotations, reflexions are forbidden and physically cannot be possible because Jacobians transformations are equal to - 1. (Gurevich, 1989). Equality to one of the determinants of linear transformation defines that, among transformations of the internal symmetry, only unimodular transformations are physically possible. Restrictions on symmetry of space-time define physical laws of conservation. The homogeneity of time defines the law of energy conservation. The homogeneity of space defines the law of impulse conservation. Isotropic spaces define the law of conservation of impulse momentum. The principle of field interaction imposes restrictions on interaction process; the interaction of particles is carried out through corresponding fields. A particle does not need to know interaction laws-it must feel a field. The law of simplicity of complex systems and the law of information conservation allow the selection of the simplest models to adequately describe the universe: the universe is identical to metagalaxy; the universe is a homogeneous object; the universe is an isotropic object; the universe is a flat object. Increase in the scale factor of inflationary expansion of the universe is $\approx 10^{45}$ times.

It was shown that the estimates of the joint entropy of matrixes mixture of electroweak interaction according to different independent experimental data are close to the estimates of the joint entropy of matrixes mixture of quarks. It testifies to the uniform information and physical nature of strong and electroweak interaction.

Taking into account Zeilinger's principle, the basic information principles of quantum mechanics construction are defined.

In particular, the joint use the physical law of energy conservation and the information law of uncertainty (information) conservation enables us to determine Hawking's formula for black holes (information spectrum of radiation) (Gurevich, 2007c).

The formula for the information spectrum of radiation of neutron stars and white dwarfs was deducted (2009). Existence of several types of substance with different dependence of information content $I$ on mass $M$ (including linear for usual substance and for dark substance $I \propto M$, square for black holes $I \propto M^{2}$, linearly-logarithmic for neutron stars and white dwarfs $I \propto M \log _{2} M$, zero for dark energy $I \equiv 0$ ) was disclosed (Gurevich, 2007c, 2009). Consumption of energy (mass) for the creation of microinformation and classical information (remembered, played back) for different types of matter was determined. In the standard model of the universe, the expansion of the mass of usual substance decreases. In the expansion of the universe with acceleration, the mass of usual substance in the beginning decreases, reaches a minimum, and then increases (Gurevich, 2007c).

Existence of optimal black holes was disclosed, and characteristics of optimal black holes (minimizing the volume of information in a part of the universe and the universe as a whole) were researched. The structure of the universe with the information minimum was determined. Limitations on the volume of information in the universe were defined (Gurevich, 2007b).

At author's approach for the estimate of information volume in a physical system, the use of holographic principle was not required. The procedure for estimating the information volume in physical objects consisted of the following. At first, the volume of information in the lower level objects - the fundamental particles (leptons and quarks) was estimated. According to Zeilinger's (1999) principle, we considered that, in the lower level objects, one bit of information was contained. Further, the volume of information in the objects of the second level was estimated. It was equal to the total of the information volume of objects of lower level plus the volume of information contained in the structure of objects of the second level of the hierarchy (mesons, baryons). The volume of information in the structure of objects of the second level is estimated on a wave function of the objects of the second level. The volume of information in objects of additional levels is similarly estimated.

Direct estimates of the information content in physical systems were given (Gurevich, 20072009). 
It is shown that the space uncertainty (information) on the particle layout in space spots the Newtonian gravitational potential (the first derivative of information on radius) and the strength of gravitational field (the second derivative of the information on radius): the type of gravitational potential is $\propto 1 / r$ (Gurevich, 2008b, 2009), the type of strength of gravitational field is $\propto 1 / r^{2}$ (Gurevich, 2008b, 2009). The same is true for the Coulomb interaction potential and the field intensity strength of the Coulomb interaction.

It was shown that, to four known types of interaction (gravitational, electromagnetic, strong, and weak); one should add one more type of interaction - information interaction (Gurevich, 2007).

The information models of cosmological objects (black holes, neutron stars, white dwarfs, and stars of solar type) were developed (Gurevich, 2007c-2009).

The information limitations on forming and merging black holes were received (Gurevich, 2008b2009).

The existence of initial discontinuities of the universe (with the use of information divergence) was proven. The estimates of the initial discontinuities mass of the universe were given (Gurevich, 2009).

Expansion of the universe from initial heterogeneity generates new heterogeneity (information). The universe expansion is the reason and source of information formation. Various physical processes in the extending universe form information (Gurevich, 1989-2009).

The curvature of the universe also generates heterogeneity (information).

It was shown that the volume of information, shaped in a frame of reference, moving with acceleration, is equal to $I=-\log _{2} J=-\log _{2} \sqrt{1-a x / c^{2}} \approx a x / c^{2}$. $J$-jacobian, $a$-acceleration, $x$ coordinate, $c$ - speed of light. We will pay attention to the analogy to the effect Unru. The appearance of thermal radiation in an accelerated frame of reference in the absence of this radiation in a counting inertial system is the appearance of additional information in an accelerated frame of reference in the absence of this information in a counting inertial system.

Estimates of the maximum volume and minimum volume, the current volume of information in the Universe was given. The estimates of the basic information characteristics of the universe were given (Gurevich, 1989 - 2009).

A statement of the universe management problem was given (Gurevich, 2007).

From the information point of view, the necessity of a physical systems description (quantum mechanics) by means of nonclassical probabilistic logic was defined (Gurevich, 2009).

It is necessary to notice, that from the principle of maximum entropy Lisi gets the necessity of the probability description of physical systems (the necessity of quantum mechanics) (Lisi, 2006).

It was shown that, in all possible universes, the informatics laws and likewise physical conservation laws operate (Gurevich, 2009).

The logic structure of nature's laws governs the stages of the universe's emergence and development. From two events in the universe's life, there is an earlier event that logically precedes the other. During the initial moments of time, information laws of nature operated. The information laws either have been set in the initial "design" of the universe or were contained in the initial heterogeneity of the universe or have been set from the outside of the universe.

The expansion of the universe from its initial heterogeneity has generated the heterogeneity (information): various types of interaction; various types of particles and fields corresponding to them; various types of atoms, molecules; various types of stars, planets; life ...

\section{Let's Look at Some of the Author's Results in More Detail}

\subsection{The Information Interaction}

The interaction of the linked (entangled) states and subsystems of the quantum system helps estimate the communication information. The linked (entangled) states, subsystems differ on the value of communication information. The unit of interaction of the linked (entangled) states and subsys- 
tems of the quantum system is the bit. Therefore, interaction of the linked (entangled) states and subsystems of quantum system is information interaction. The interaction of the linked (entangled) states and subsystems does not depend on their layout in space or the distance between them. It follows from an information conservation law of uncertainty (information). At a change of coordinates, orientation in space separate q-bits and subsets q-bits, and the linked (entangled) state as a whole uncertainty (information) of interaction is saved.

It is possible to translocate q-bits, parts of the linked (entangled) subsystems, with any velocity from each other, saving the value of information interaction.

The maximum information interaction between subsystems $\mathrm{A}$ and $\mathrm{B}$ of systems $A+B$ is determined by the volume of uncertainty (information) in subsystems and is equal to $I_{A B \max }=\log _{2} d$, where $d$ is the dimension of the subsystems.

It is necessary to add to the four known types of interaction (gravitational, electromagnetic, strong, weak) one more type of interaction - the information interaction (Gurevich, 2007c).

\subsection{Estimations of Volume of the Information in the Systems Consisting of $n$ of Elementary Systems (q-bits)}

Gurevich (2007) shows that the physical system can be represented as a direct sum of direct products of the q-bits. Therefore, it is necessary to assess the volume of information in q-bits systems. Zeilinger (1999) proposed the Foundational Principle for Quantum Mechanics: An elementary system carries 1 bit of information. Let's estimate the volume of information in the system consisting of $n$ elementary systems ( $n$ q-bits). In the beginning, we shall consider systems with equiprobable basic states.

Let us assume that a system consists of noninteracting $n$ q-bits. The q-bit is described by the wave function $\psi=\frac{1}{\sqrt{2}}(|0\rangle+|1\rangle)$. Here $|0\rangle,|1\rangle$ are basic states of the q-bit. Measurements of the qbit states will give the states $|0\rangle$ and $|1\rangle$ with probabilities equal to $\frac{1}{2}$. Uncertainty $N_{1}$ (information $I_{1}$ ) of the q-bit is equal to 1 bit $N_{1}=I_{1}=H_{1}=-\left(\frac{1}{2} \log _{2} \frac{1}{2}+\frac{1}{2} \log _{2} \frac{1}{2}\right)=1$. Here $H$ is the information entropy (Shannon, 1948). Hence, in the system consisting of $n$, not cooperating q-bits with equiprobable basic states that the volume of information $I_{n}$ is proportional to the number of q-bits $n$ and equal to $n$ bits. This determines the minimal volume of information in system of $n$ q-bits with the equiprobable basic states. This explains the linear dependence of the volume of information on mass in usual substances (in fundamental particles - quarks, leptons, photons).

Let us assume that a system consists of $n$ q-bits interacting in pairs with equiprobable states and is described by the following wave function $\psi_{n}=\frac{1}{\sqrt{2}}\left(\left|0_{1}\right\rangle\left|0_{2}\right\rangle \ldots\left|0_{n}\right\rangle+\left|1_{1}\right\rangle\left|1_{2}\right\rangle \ldots\left|1_{n}\right\rangle\right)$. Every qbit $i$ has the wave function $\psi_{i}=\frac{1}{\sqrt{2}}\left(\left|0_{i}\right\rangle+\left|1_{i}\right\rangle\right) \cdot\left(\left|0_{i}\right\rangle,\left|1_{i}\right\rangle\right.$ are basic states of the $i$-th q-bit. The information of communication $I_{i j}=H_{i}+H_{j}-H_{i j}$ (Gurevich, 2006a) of the pair of interacting q-bit $i j$ described by the wave function $\psi_{i j}=\frac{1}{\sqrt{2}}\left(\left|0_{i}\right\rangle\left|0_{j}\right\rangle+\left|1_{i}\right\rangle\left|1_{j}\right\rangle\right.$ ) is equal to 1 bit (Gurevich, 2006a). The volume of the information of communication in the system consisting of $n$ q-bits interacting in pairs with equiprobable basic states is equal to $I_{n \text { com }}=\frac{n \cdot(n-1)}{2}$ bits. The total volume 
of information $I_{n}$ in the system containing $n$ q-bits interacting in pairs with equiprobable basic states, is equal to $I_{n}=n+\frac{n \cdot(n-1)}{2}=\frac{n \cdot(n+1)}{2}$ bits. This determines the maximal volume of information in the system consisting of $n$ q-bits. At $n>>1$ the volume of information in the system of $n$ q-bits interacting in pairs is equal to $I_{n} \approx \frac{n^{2}}{2}$. This explains the quadratic dependence of the volume of information on the mass in black holes. The system consisting of $n$ in pairs interacting q-bits contains $n$ bits more information than the system consisting of $n-1$ in pairs interacting qbits: $I_{n}-I_{n-1}=\frac{n \cdot(n+1)}{2}-\frac{(n-1) \cdot n}{2}=n$.

Let us assume that the system consists of $n$ q-bits, where they make groups of $k$ q-bits and each of the q-bits interacts only with q-bits of the group (we consider that $n$ divided by $k$ ). The volume of information in groups consisting of $k$ in pairs interacting q-bits with equiprobable basic states is equal to $\frac{k \cdot(k+1)}{2}$ bits. Hence, in the system of $n$ q-bits with limited interaction there are $I_{n / k}=\frac{n}{k} \frac{k \cdot(k+1)}{2}=\frac{n \cdot(k+1)}{2}$ bits. This explains the linear dependence of the volume of information on mass of composite particles in usual substance (for example, in elementary particles baryons, mesons and also atoms).

In general the volume of information $I_{n}$ in the system consisting of $n$ q-bits with equiprobable basic states, is not less than $n$ bits and no more than $\frac{n \cdot(n+1)}{2}$ bits.

\subsection{Information Estimate of the Mass of Initial Discontinuities of Universe}

The results obtained with the help of information considerations lead to the following basic conclusions: the universe, at the extension, generates discontinuities (information) from initial discontinuities (information) (Dolgov et al.,1988); we showed that, at the initial time, the heterogeneity of usual substance matter and dark substance in the universe exist; we show that, at the initial time, the heterogeneity of dark energy in the universe did not exist; the laws of nature and physical laws completely determine that the development, properties, and characteristics of the universe must be contained in the initial heterogeneity of our universe. Physical laws operate in our universe from the moment of time of not less than $t=1 E-44 \mathrm{~s}$.

For a record, fixing the natural laws and physical laws, the certain volume of classic information $I_{p f}$ is needed. Consequently, a volume of the classic information $I_{h t y}$ in the initial heterogeneity of universe must be not less than the volume of the classic information is in the natural laws and physical laws $I_{g t y}<I_{p f}$

The mass of the initial heterogeneity is more then $1 \mathrm{E}+15^{*} l_{p f}{ }^{*} k^{*} T / c^{\wedge} 2$, or the mass of the initial heterogeneity is proportional to the volume of the classical information in the physical laws and is inversely proportional to the square root of the time of life of our universe and inversely proportional to the square of the speed of light.

The mass of the initial heterogeneity of the universe required to memorize or store the physical laws $(1 E+7$ classical bits) at the temperature of the universe $1 E+12 K$ (the lifetime of the universe $1 E-5 \mathrm{~s}$ ) is about $1 E-8 \mathrm{~kg}$ (about one Planck's mass).

To get the $1 E+7$ classical bits of information at the time $t=E-34 s$, with the Fridman's expansion of the universe, it needs approximately $1 \mathrm{E}+5$ classic bits at the time $\mathrm{t}=\mathrm{E}-44 \mathrm{~s}$ or a mass of the initial heterogeneity of the universe of approximately $1 \mathrm{E}+12 \mathrm{~kg}$. At the sedate extension of the uni- 
verse from $1 \mathrm{E}-34 \mathrm{~s}$ to $1 \mathrm{E}-10 \mathrm{~s}$ from 1 bit of the classical information 160 bits of the classical information were formed. Therefore, for deriving $1 \mathrm{E}+7$ bits classical information at the moment of $1 \mathrm{E}-10$ $s$ it is necessary to have $1 \mathrm{E}+5$ bits classical bits at the moment of $1 \mathrm{E}-34 \mathrm{~s}$ or approximately the mass $1 \mathrm{E}+7 \mathrm{~kg}$. This, apparently, is impossible. Therefore, the initial information is assumed to be generated, appreciably, at the inflationary extension of the universe.

At the inflationary extension of the universe from $1 \mathrm{E}-34 \mathrm{~s}$ to $1 \mathrm{E}-32 \mathrm{~s}$, from one bit of the classical information containing in initial heterogeneities of the universe $1 \mathrm{E}+3$ bits of the classical information are shaped. At the inflationary extension from $1 \mathrm{E}-34 \mathrm{~s}$ to $1 \mathrm{E}-32 \mathrm{~s}$ and the further sedate extension of the universe from $1 \mathrm{E}-32 \mathrm{~s}$ to $1 \mathrm{E}-10 \mathrm{~s}$ from one classical information bit $1 \mathrm{E}+5$ bits classical information are shaped. For deriving $1 \mathrm{E}+5$ bits classical information at the moment of $1 \mathrm{E}-10 \mathrm{~s}$, it is necessary to have about $1 \mathrm{E}+5$ classical bits at the moment of $1 \mathrm{E}-34 \mathrm{~s}$ or the mass $1 \mathrm{E}+4 \mathrm{~kg}$. This is an estimate of the mass of initial heterogeneity of the universe at the moment of $1 \mathrm{E}-34 \mathrm{~s}$, the mass that is necessary for containing (storing) the physical laws of nature.

It testifies in favor of the improved hypothesis to the initial information (Leskov, 2006): “... At the

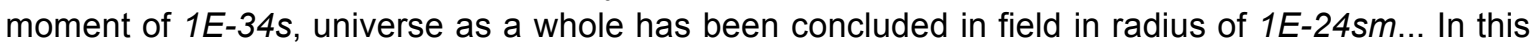
size already there was heterogeneity of mass of the order $1 E+4 \mathrm{~kg}$ from which all information on the universe future was generated..."

\subsection{Information Volume in Our Universe}

Information is inseparably linked with matter and energy. Information is physical heterogeneity steady for certain time, heterogeneity of matter and energy (Gurevich, 1989-2009). The energy necessary for the formation of one bit of microinformation (Chernavsky, 2002) is equal to $E_{b i t}=k T \ln 2$ (Brillouin, 1960). The mass necessary for formation of one bit of the microinformation is equal to $m_{b i t}=k T \ln 2 / c^{2}$. The values of energy and mass of the carrier of 1 bit of microinformation are resulted at temperatures $3 \mathrm{~K}(300 \mathrm{~K})$. The minimum energy for 1 bit is equal to $4,141 \mathrm{E}-23$ $(4,141 \mathrm{E}-21)$ joule. The minimum mass for 1 bit is equal to $4,608 \mathrm{E}-40(4,608 \mathrm{E}-38) \mathrm{kg}$. On the average in atoms for 1 bit of information is used $\approx 1,69 \cdot 10^{-28} \mathrm{~kg}$ of mass of substance (for example, in atom of hydrogen is used $\approx 1,6 \cdot 10^{-28} \mathrm{~kg}$, in atom of lithium $-\approx 1,93 \cdot 10^{-28} \mathrm{~kg}$ ). It is approximately by $\approx 10^{12}$ times more than the mass necessary for formation of one bit of microinformation.

The reason and source of information formation is expansion of the universe and initial heterogeneity. At symmetry infringement between weak and electromagnetic interactions in the universe, it is formed $10^{90}$ bits. The information mechanism of particles formation in the inflationary universe generates quantity of particles, comparable with the standard estimation of the number of particles in the universe, - an order of $10^{80}-10^{90}$.

The minimum possible volume of information in the universe with prevalence of substance is $\approx 1,7 \cdot 10^{79}$ in the universe with prevalence of radiation is $\approx 10^{91}$ bits. The greatest possible volume of information in the universe is $\approx 10^{120}$ bits (Gurevich, 1989-2009). Growth of volume of information at sedate expansion of the universe is $\propto \log _{2} t$. Reduction of density of information at sedate expansion of the universe is $\propto\left(\log _{2} t\right) / t^{2}$. The growth of information volume at inflation expansion of the universe is $\alpha \alpha t$. The reduction of density of information at inflation expansion of the universe is $\propto t e^{-3 \alpha t}$.

\subsubsection{Information Volume in Some Fundamental, Elementary Particles and Atoms}

Fundamental particles are the simplest physical systems (elementary systems by Zeilinger, 1999). 
There is 1 bit in a lepton. There is 1 bit in a quark. One photon with circular polarization contains 1 bit. One photon, $Z^{0}$-bozone-products of electroweak interaction-contains 0,78 bits.

Elementary particles represent physical systems of the second level of complexity.

There are 9,422 bits in a proton, a neutron (taking into account the structure of protons and neutrons, the information in quarks, and the colors of quarks).

Atoms represent physical systems of the third level of complexity.

There are 11,422 bits in the atom of hydrogen (taking into account the structure of atom, the information in protons and electrons). There are 39,688 bits in the atom of helium. ... There are 109,642 bits in the atom of carbon. ... There are 544,21 bits in the atom of iron (26th element). There are 2334,436 bits in the atom of uranium, ... In the above-mentioned cases the structure of atoms and external uncertainty of electrons are not considered.

The estimates of the joint entropy of matrixes mixture of electroweak interaction $(1,7849 ; 1,7787$; $1,7645 ; 1,7945)$, according to different independent experimental data, are close to the estimates of the joint entropy of matrixes mixture of quarks $(1,7842,1,7849)$ (Gurevich 2008, 2009).

\subsubsection{Information Volume in Stars}

The sun contains $\approx 1,3 \cdot 10^{58}$ bits. The white dwarf of solar mass contains $\approx 1,24 \cdot 10^{59}$ bits. The neutron star of solar mass contains $\approx 2,38 \cdot 10^{59}$ bits.

\subsubsection{Information Volume in Black Holes}

The existence of matter of two types - with square-law and with linear dependence of information volume on mass - is the source, the reason of existence, of optimal black holes which minimize the volume of information in any region of the universe and in the universe as a whole.

The information volume and the mass of optimal black holes, that are yielded by deciding the direct problem (minimization of volume of information in the system (usual substance - black hole) for a given mass of the system) and the dual problem (maximization of mass of the system (usual substance - black hole) for a given volume of information in the system) respectively, coincide.

There are $\approx 10^{62}$ bits in the optimal black hole generated in the system «radiation (photons) black hole» at the temperature of radiation $-2,7 \mathrm{~K}$. There are $\approx 2,57 \cdot 10^{38}$ bits in the optimal black hole generated in the system «hydrogen (protons) - black hole».

At the temperature of radiation $T=m_{p} c^{2} / 9,422 k \ln 2=1,555 \cdot \mathrm{E}+12 \mathrm{~K}$ (at the time from «the Big

Bang» of the universe $10^{-5}$ ), the mass of the optimal black holes that have arisen in the systems «radiation - black holes» is equal to the mass of the optimal black holes which have arisen in the systems «hydrogen (protons) - black hole».

In masses of the optimum black holes shaped from various types of atoms of ordinary substances or a mixture of various types of atoms of ordinary substances, information contents are approximately identical.

The black holes of solar mass contain $\approx 7,72 \cdot 10^{76}$ bits. The black holes in centers of galaxies contain $\approx 10^{90}-10^{107}$ bits. 


\subsubsection{Information Volume in Galaxies}

In galaxies having $10^{11}$ stars, there are about $10^{69}$ bits. In galaxies having $10^{11}$ of stars and containing, in kernels, super massive black holes with the mass of $\approx 10^{6}-10^{10}$ of solar mass, there are $\approx 10^{99}-10^{107}$ bits.

\subsubsection{Information Dependence of Temperature of Radiation on Mass}

For a black hole, the dependence of temperature on mass (Hawking's spectrum) looks like $T=\left(\hbar c^{3} \ln 2\right) /(4 \pi G M k)$. For a neutron star, the dependence of temperature on mass (an information spectrum) looks like $T=\left(m_{n} c^{2}\right) / k\left(9,422+\log _{2} M / m_{n}\right)$.

\subsubsection{Information Restrictions at Creation of Black Holes from Stars}

The mass of the black hole formed from the star of the sun's type is no more than $\approx 8 \cdot 10^{20} \mathrm{~kg}$.

The mass of the black hole formed from the white dwarf of solar mass is no more than $\approx 2,5 \cdot 10^{21} \mathrm{~kg}$. The mass of the black hole formed from the neutron star of solar mass is no more than $\approx 4,17 \cdot 10^{21} \mathrm{~kg}$.

Note: The black hole at formation uses only part of mass. Other mass, in the form of usual substance, dissipates in surrounding space, and other objects can be formed of it.

\subsubsection{Information Restrictions at the Merging of Black Holes}

At the merging of two black holes with the mass $M_{1} M_{2}$, without the use of additional usual substance, the mass of the resulting black hole is less, than $\sqrt{M_{1}^{2}+M_{2}^{2}}$.

At the merging of two black holes with the mass $M_{1} M_{2}$, with the use of additional usual substance, the mass of the resulting black hole is more than $\sqrt{M_{1}^{2}+M_{2}^{2}}$.

\subsubsection{Classical Information}

Nitrogenous basis contains $\log _{2} 4=2$ bits of the classical information (macroinformation).

Amino acids contain $\log _{2} 20=4,32$ bits of the classical information. For 1 bit of information formed by amino acids and nitrogenous basis, $4,43 \mathrm{E}-25$ and $1,05 \mathrm{E}-25 \mathrm{~kg}$ of mass are needed. Redundancy of classical information formed by life, in relation to micro information, at the temperature of $300 \mathrm{~K}$ is greater by a factor of $\approx 10^{13}$. Proteins and DNA for formation of 1 bit of information use mass by three orders more than atoms. Hence, life is an effective way of forming classical information. Proteins of yeast contain about 2,000 bits of classical information. One chromosome of a person contains $(1-5) \cdot 10^{8}$ bits of classical information. One person contains $\approx 10^{26}$ bits of classical information. Biomass of the Earth contains about $10^{40}$ bits of classical information. If $100 \%$ of the Earth's mass is used for the formation of live substance, it will generate about $10^{50}$ bits of classical information. If $1 \%$ of the universe's mass is used for the formation of live substance, it will generate approximately $10^{75}$ bits of classical information. $10^{40}-10^{77}$ bits is a range of possible volume of classical information in the universe defined by the data known now. The volume of classical infor- 
mation formed by terrestrial civilization is $<10^{30}$ bits/year. Parity of volumes of information in the universe in a year, generated by matter and civilization is $\approx 10^{-49}$. The share of information formed by civilization on one star system is equal to $10^{-27}$. It shows that the contribution of terrestrial civilization to information formation of the universe is now insignificant.

\subsubsection{Fundamental Limits on Information Capacity Storage Devices}

Regarding estimates of the information volume in atoms, amino acids, nitrogenous bases, the differential information capacity of a substance determines the fundamental limits of information capacity storage devices. The differential capacity information of storage devices, based on a combinations of atoms, does not exceed $\approx 10^{-25} \mathrm{bits} / \mathrm{kg}$, and the information capacity of the storage mass $1 \mathrm{~kg} \leq 10^{25}$ bits; it can be enhanced with respect to the current level of no more than $\approx 10^{11}$ times. The differential information capacity of storage devices, built on the basis of atoms, does not exceed $\approx 10^{-28}$ bits $/ \mathrm{kg}$, and the information capacity of the storage mass $1 \mathrm{~kg} \leq 10^{28}$ bits; it can be increased by no more than $10^{14}$ times.

\subsubsection{Fundamental Restrictions on Productivity of the Information Systems}

The difference between the energies of the basis states of the hydrogen atom, considered as a qbit, impose fundamental limitations on the speed of computing devices. The number of operations a hydrogen atom can perform as a q-bit is limited to $k_{o p / s}=2 \Delta E / \hbar \approx 1,5^{*} 10^{12}$ operations per second.

The productivity of a computer built from atoms of hydrogen, whose mass is one $\mathrm{kg}$, is not more than $10^{39} \mathrm{op} / \mathrm{sec}$. Given the restrictions of $10^{28} \mathrm{bit} / \mathrm{kg}$ and $1,5^{*} 10^{12} \mathrm{op} / \mathrm{sec}$, you can add a number of fundamental natural limits, such as the speed of light, the elementary charge, Planck's time, and so on.

\subsubsection{Cognitive Process of the Universe}

The universe, the information volume of which is finite, is effective and completely knowable (Gurevich 2006c).

The subject of the cognitive process is a classical object (for example, terrestrial civilization).

In the course of the universe's cognitive process, compression of information is not less than $\approx 10^{20}$ times and no more than $\approx 10^{76}$ times. The gravitation law, in particular, compresses the information than by no less of a factor than $4 \cdot 10^{183}$.

Interpretation of cognitive process by methods of quantum mechanics (the description and measurement) on the basis of information parities is possible. The knowledge is carried out through a hypothetical information channel - "the knowledge channel of nature." The limited throughput of "the knowledge channel of nature" defines as impossible "exact" (in classical sense) descriptions and measurements of quantum objects. To increase accuracy (uncertainty) of the description/measurement of one of the components, the observer is compelled to reduce accuracy (uncertainty) of the description/measurement of the other.

\subsubsection{Information Unity of All Possible Universes}

As heterogeneity should exist in the universes with any physical laws, the approach that is based on information properties of heterogeneities of any nature and corresponding information laws and restrictions, and also physical laws of conservation following from them, extends on all possible universes. Thereby, physical laws of conservation and information restrictions on other possible 
physical laws in different universes are identical. Does it mean that all possible universes are identical?

\section{The Approach 2. (Verlinde E. 2010)}

There are many works that appeared in the beginning of 2010. Among them was Verlinde (2010). The starting position is: the first law of thermodynamics, the second law of thermodynamics, holographic principle, Hawking's formula, and Unru effect.

For information, these aspects are noted: the law of gravitation (2009-2010), Newton's second law (Verlinde, 2010; Makela, 2010; Smolin, 2010), Fridman's equations (Rong-Gen at al., 2010), and the irreversibility of time (Maccone, 2009).

\section{Conclusion}

The works of the author and American, Canadian, European, Chinese ... scientists are confirming the primacy of information laws; information laws (informatics laws) define and restrict physical laws; informatics laws have a general, universal character and operate in all possible universes, even in universes with different physical laws. The given data shows that the priority use of information methods of physical systems research belongs to the author, though the final results of foreign scientists are very interesting and important. Informatics laws, together with physical laws, will allow us to open all secrets of nature, in particular, to construct a theory of quantum gravitation.

\section{References}

Ashby W. (1956). An Introduction to Cybernetics. London: Chapman \& Hall.

Brillouin, L. (1960). Science and Information Theory. Moscow: Fizmatgiz.

Chernavsky, D.S. (2002). Synergetics and Information. Moscow: Nauka.

Dolgov, A.D., Zeldovich, J.A.B., \& Sazhin, M.V. (1988). Cosmology of the Early Universe. Moscow: Publishing House of the Moscow University.

Einstein, A., Podolsky, B., \& Rosen, N. (1935). Can Quantum-Mechanical Description of Physical Reality Be Considered Complete? Physical Review, 47(10), 777-780.

Gödel K. (1931). "Über Formal Unentscheidbare Sätze der Principia Mathematica und Verwandter Systeme, I." Monatshefte für Mathematik und Physik 38, 173-198.

Gurevich, I.M. (1989). Law of Informatics: A Basis of Researches and Designing of Complex Communication and Management Systems. Moscow: Ecos.

Gurevich, I.M. (2003). Informatics Laws: A Basis of a Structure and Cognitive of Complex Systems. Moscow: Antikva.

Gurevich, I.M. (2006a). Information Characteristics of the Entangled States of the q-bits. Information Technology 5, 32-39.

Gurevich, I.M. (2006b). The Information Estimation of "Force" of Interaction the Entangled States. Proceedings of The International Scientific Conference "Problems of Cybernetics and Informatics." Baku, Azerbaijan.

Gurevich, I.M. (2006c). Data Compression "Intelligence" in the Process of Cognition of the Universe. The Bulletin of the Special Astrophysical Observatory of the Russian Academy of Sciences 60-61, 145-157.

Gurevich, I.M. (2007a). Information Model of a Black Hole. Proceedings from BAK '07. Kazan, Russia.

Gurevich, I.M. (2007b). About Restrictions on Volume of the Information in the Universe. Paper presented at the 58th International Astronautical Congress, 2007.

Gurevich, I.M. (2007c). Informatics Laws: A Basis of a Structure and Cognitive of Complex Systems (2nd ed.). Moscow: Torus Press.

Gurevich, I.M. (2008a). Estimation of Information Volume in Cosmology Objects. Proceedings from The Second International Conference "Problems of Cybernetics and Informatics." Vol. 2. (pp. 128-131). Baku: Azerbaijan.

Gurevich, I.M. (2008b). Estimation of the Basic Information Characteristics of the Universe. Information Technologies 12 Appendix $32 \mathrm{p}$.

Gurevich, I.M. (2009). The Information Characteristics of Physical Systems. 11 FORMAT. Moscow, Cypress. Sevastopol, $170 \mathrm{p}$.

Gurevich, I.M. (2010). The Main Information Characteristics of Physical, Chemical and Biological Systems. Modern Trends in Theoretical and Applied Biophysics, Physics and Chemistry. BPPC - 2010. Vol. 1. In Common Questions of Physics and Chemistry: Materials of VI International Science-Technical Conference, SevNTU (pp. 83-86). Sevastopol.

Leskov, S. (2006). Let's Find a Place for the God. Retrieved from http://www.inauka.ru/analysis/article60920.html. 
Lloyd, S. (2001). Computational Capacity of the Universe. Retrieved from http://arxiv.org/abs/quant-ph/0110141 Lisi, A. (2006). Quantum Mechanics from a Universal Action Reservoir. Retrieved from http://arxiv.org/pdf/physics/0605068 Maccone, L. (2009). A Quantum Solution to the Arrow-of-Time Dilemma. Retrieved from http://arxiv.org/pdf/0802.0438 Mäkelä, J. (2010). Notes Concerning "On the Origin of Gravity and the Laws of Newton" by E. Verlinde. Retrieved from http://arxiv.org/abs/1001.3808

Neumann, J. (1932). Mathematical Bases of Quantum Mechanics. Berlin: Verlag von Julius Springer.

Penrose, R. (1989). The Emperor's New Mind. Oxford: Oxford University Press.

Rong-Gen, C., Li-Ming, C., \& Nobuyoshi, O. (2010). Friedmann Equations from Entropic Force. Retrieved from http://128.84.158.119/pdf/1001.3470v1

Shannon C. A. (1948). Mathematical Theory of Communication. Bell System Technical Journal, 27, 379-423, 623-656.

Smolin, L. (2010). Newtonian Gravity in Loop Quantum Gravity. Retrieved from http://arxiv.org/abs/1001.3668

Verlinde, E. (2010). On the Origin of Gravity and the Laws of Newton. Retrieved from http://arxiv.org/abs/1001.0785

Wheeler, J.A. (1998). Geons, Black Holes \& Quantum Foam: A Life in Physics. New York NY: W.W. Norton \& Company.

Zeilinger, A. (1999). A Foundational Principle for Quantum Mechanics. Foundations of Physics, 29(4), 631-643.

\section{About the Author}

Igor Gurevich

is a Senior Researcher at the Institute of Informatics Problems of the Russian Academy of Sciences; Doctor of Technical Sciences; the main designer for Hetnet Consulting Corp. Moscow, Russia; and a teacher in the Moscow Physical-Technical Institute. Scientific interests: Network of classical and quantum computers; Theoretical Informatics, Physical Informatics. 